\title{
Polydopamine decorated 3D nickel foam for extraction of sixteen polycyclic aromatic hydrocarbons
}

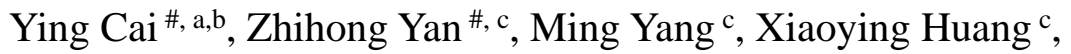

Weiping Min ${ }^{\mathrm{b}}$, Lijia Wanga, Qingyun Cai ${ }^{\mathrm{a}, *}$

\begin{abstract}
In this work, polydopamine coated 3D nickel foam (NF-PDA) was prepared and applied as sorbent for the solid phase extraction (SPE) of 16 polycyclic aromatic hydrocarbons (PAHs) from water samples. NF-PDA were synthesized by situ oxidative self-polymerization procedure and characterized by using the techniques of scanning electron microscopy (SEM), energy dispersive spectrum analysis (EDS) and X-ray photoelectron spectroscopy (XPS). Its performance was evaluated by the SPE of 16 PAHs from water samples, followed by gas chromatographymass spectrometrical (GC-MS) analysis. The effects of the main experimental parameters (i.e. sorbent amount, desorption solvent, extraction time, water sample volume, elution volume, elution time, ionic strength and samle solution $\mathrm{pH}$.) that could affect the extraction efficiencies were investigated. The results demonstrated that the NF-PDA had an excellent adsorption capability for the compounds. The methodology was validated for river water and wastewater, obtaining recoveries ranging from 89.6 to $97.5 \%$ with relative standard deviation values lower than $7.3 \%$ and limits of detection in the range $2.3-16.5 \mathrm{ng} / \mathrm{L}$.
\end{abstract} Keywords: Nickel foam; Polycyclic aromatic hydrocarbons; Gas chromatography-mass spectrometry; green chemistry.

\footnotetext{
\# These authors contributed equally to this study and share first authorship.

*Corresponding author. Tel.: +86-73188821848.

E-mail: qycai0002@gmail.com, qycai0001@hnu.edu.cn (Q.Cai).
} 


\section{Introduction}

Polycyclic aromatic hydrocarbons (PAHs) are an important class of organic pollutants and their primary source in the environment is from the incomplete combustion of carbonaceous materials [1]. PAHs are of great concern because of their widespread occurrence and toxic effects on ecosystem and human health [2,3]. Sixteen PAHs are included in the priority pollutants list of the US Environmental Protection Agency (US EPA), namely naphthalene (Nap), acenaphthylene (Aceny), acenaphthene (Acen), fluorene (Flu), phenanthrene (Phen), anthracene (Ant), fluoranthene (Fluo), pyrene (Pyr), chrysene (Chr), benz[a]anthracene $(\mathrm{B}[\mathrm{a}] \mathrm{A})$, benzo[b]fluoranthene $(\mathrm{B}[\mathrm{b}] \mathrm{F})$, benzo[k]fluoranthene $(\mathrm{B}[\mathrm{k}] \mathrm{F})$, benzo[a]pyrene $(\mathrm{B}[\mathrm{a}] \mathrm{P})$, dibenz[a,h]anthracene $(\mathrm{DB}[\mathrm{a}, \mathrm{h}] \mathrm{A})$, indeno[1,2,3cd]pyrene $(\mathrm{I}[1,2,3-\mathrm{cd}] \mathrm{P})$ and benzo[ghi]perylene $(\mathrm{B}$ [ghi]P) [4]. PAHs are semivolatile organic compounds that can exist in almost all the environmental medium [5]. The pathways by which PAHs enter the surface water bodies include atmospheric deposition, urban runoff, municipal effluents, industrial effluents, and oil spillage or leakage [6]. So it's very important to detect the polycyclic aromatic hydrocarbons in the waters.

Presently the two most frequently employed techniques to determine PAHs are HPLC with fluorescence, UV, or diode array detection $[7,8]$ and GC with MS detection [7,9-11]. Besides fluorescence detector which used in combination with HPLC can operate selectively through choosing proper wavelengths, the HPLC methods are heavy dependence on chromatographic retention time for compound identification. But peak identification based solely on retention time is subject to interference from other components, making trace level PAH contamination difficult to characterize. GC-MS technique has become established as the accepted method for PAH determination in the environment for over 15 years [10]. Additionally, the HPLC methods are typically an order of magnitude lower in sensitivity than GC-MS [12].

Before instrumental analysis, it is necessary to extract PAHs from samples because PAH levels in environmental water samples are usually very low for their very low water solubility and high hydrophobicity. . Very recently, some new "green" and effective sample extraction methods have been developed, such as solid-phase microextraction [13-15], microsolid phase extraction [16-18] and magnetic solid-phase extraction [19-23]. In short, each technique has its own virtues and drawbacks. Effective though these methods are, there are some drawbacks existed, such as excessively complex preparation procedure, expensive reagents, low extraction efficiency, strong dependence on certain extraction condition, and co-eluted compounds that interfere with determination of analytes. New sorbents with simple preparation process, low price and 
high adsorption efficiency should be developed for environmental analysis.

Herein, for the first time, we introduced foam metal as new absorbing material. Foam metal is a 3D cellular material, and the base architecture of which consists of a periodic array of hollow tubes that connect at nodes, forming an octahedral unit cell without any lattice members in the basal plane [24]. Its 3D nanostructure array architecture can offer not only a large specific surface area, but also an improved path for ion diffusion and electron transportation [25]. Up to now, foam metal is mainly used for materials of high-performance supercapacitors [26], and there is rarely report on foam metal applying in SPE.

This paper reports the exploration of the suitability of sorbents based on polydopamine decorated 3D nickel foam (NF-PDA) for the development of simple, robust and reliable sample preparation methods for the determination of PAHs in water samples. Dopamine is a catecholamine neurotransmitter mimic of the adhesive proteins which has attracted wide interest due to its self-polymerization capacity in aqueous phase under weak alkaline conditions [27]. PDA also has good environmental stability and biocompatibility, and polydopamine coatings can serve as a versatile platform for secondary surface-mediated reactions, which has attracted the interest of researchers [28]. Recently, PDA has been shown to be a good decorating material to enhance extraction capability. It is reported that, polydopamine-coated magnetic nanoparticles have been successfully synthesized and developed as a adsorbent for enrichment of PAHs [29,30] and estrogenic compounds [31,32], while polydopamine coated stainless steel wire was used for solid-phase microextraction of PAHs [33].

In this work, NF-PDA was synthesized by situ oxidative selfpolymerization procedure. The cellular structure of NF make the sorbent has large contact area. Polydopamine coat can promote to extract PAHs through hydrophobic interactions, as well as $\pi-\pi$ interactions from the catechol structure. The parameters affecting extraction efficiencies and concentration factors were optimized. The analytical characteristics of the methods developed were established and the methods were successfully applied to the determination of PAHs in water samples. The SPE method based on NF-PDA avoids the time-consuming step of column loading or pressure filtration and possesses great potential in pretreatment of water samples.

\section{Experimental}

\subsection{Reagents and materials}


Tris(hydroxymethyl)-amino methane (Tris, 99\%) and dopamine hydrochloride (dopamine $\mathrm{HCl}, 98 \%$ ) were purchased from Macklin Biochemical Co., Ltd (China). HPLC-grade methanol, n-hexane and acetonitrile (99\%) were bought from Merck (Darmstadt, Germany). Other chemicals such as $\mathrm{NaCl}, \mathrm{HCl}$ and $\mathrm{NaOH}$ were of analytical grade and used without further purification. A piece of Nickel foam (purity: $>99.5 \%, 1.0 \mathrm{~mm}$ thickness, Projected area: $30 \mathrm{~mm} \times 20 \mathrm{~mm}$ ), was obtained from the Kunshan Jiayisheng Electronic Technology Co., Ltd.

610/8100 PAH standard solution was bought from ANPEL Laboratory Technologies (Shanghai) Inc. The PAH standard solution contains Nap, Aceny, Acen, Flu, Phen, Ant, Fluo, Pyr, Chr, B[a]A, B[b]F, $\mathrm{B}[\mathrm{k}] \mathrm{F}, \mathrm{B}[\mathrm{a}] \mathrm{P}, \mathrm{DB}[\mathrm{a}, \mathrm{h}] \mathrm{A}, \mathrm{I}[1,2,3-\mathrm{cd}] \mathrm{P}$ and $\mathrm{B}[\mathrm{ghi}] \mathrm{P}$, each at $2 \mathrm{mg} / \mathrm{mL}$ in benzene/methylene dichloride $(1 / 1, \mathrm{v} / \mathrm{v})$. The PAH stock solution was prepared in methanol at a concentration of each at $2 \mu \mathrm{g} / \mathrm{mL}$, and kept at 4 ${ }^{\circ} \mathrm{C}$ in darkness. PAH working solutions were prepared by proper dilution of the stock solution.

River water and waste water were used for real sample investigation. River water sample was collected from the Ganjiang River in Nanchang (Jiangxi, China) and collected at $10 \mathrm{~cm}$ depth below the water surface. Waste water sample was collected from water treatment plants effluents (Jiangxi, China). Water samples were collected on Oct. 2015, and were analyzed within $24 \mathrm{~h}$.

2.2. Instrumentations and analytical conditions

The size and morphological characterization of the particles were observed by scanning electron microscopy (SEM, FEI Quanta250, FEI, USA). Energy dispersive spectroscopy (EDX) mapping of the samples was undertaken using an Oxford X-Act EDX attachment (Oxford instruments, UK). X-ray photoelectron spectroscopy (XPS) was tested on a Thermo ESCALAB 250Xi X-ray photoelectron spectrometer (Thermo Scientific, USA). A nitrogen sorption experiment was carried out using a SSA-4300 specific surface area (Builder, China).

Analyses were conducted using an Agilent 7890A GC (Agilent Technologies, USA) system coupled to an Agilent 5975C mass 
spectrometer (Agilent Technologies, USA). Helium (purity 99.999\%) was employed as the carrier gas at a flow rate of $1.8 \mathrm{~mL} / \mathrm{min}$. Samples $(1 \mu \mathrm{L})$ were injected in splitless mode. The injector temperature was set at 280 ${ }^{\circ} \mathrm{C}$ and the interface temperature maintained at $280{ }^{\circ} \mathrm{C}$. The chromatographic separation of the PAHs was performed on a $30 \mathrm{~m} \times 0.25$ $\mathrm{mm} \times 0.25 \mu \mathrm{m}$ HP-5 capillary column (Agilent). The GC oven was initially held at $80{ }^{\circ} \mathrm{C}$ for $1 \mathrm{~min}$ and increased to $250{ }^{\circ} \mathrm{C}$ at $25^{\circ} \mathrm{C} / \mathrm{min}$ and held for $6 \mathrm{~min}$, then increased to $300^{\circ} \mathrm{C}$ at $10{ }^{\circ} \mathrm{C} / \mathrm{min}$ and held for $2 \mathrm{~min}$. Preliminarily, full scan electron ionization data were acquired to determine appropriate masses under the following conditions: ionization energy: $70 \mathrm{eV}$, mass range: 35-350 amu, scan time: 3 scan/s. PAH standards and samples were finally analyzed in selective ion monitoring (SIM) mode for quantitative determination of the analytes. The masses monitored were as follows: 3-5 min, m/z 128, 127, 102 for Nap; 5-6 min, m/z 152, 154 for Aceny and Acen; 6-7 min, m/z 166, 165 for Flu; 7-8 $\mathrm{min}, \mathrm{m} / \mathrm{z} 178$ for Phen and Ant; 8-10 min, m/z 202, 203, 200 for Fluo and Pyr; 10-13 min, m/z 228, 226, for Chr and B[a]A; $13-18 \mathrm{~min}, \mathrm{~m} / \mathrm{z} 252$ for $\mathrm{B}[\mathrm{b}] \mathrm{F}, \mathrm{B}[\mathrm{k}] \mathrm{F}$ and B[a]P; 18-22 min, m/z 276, 278 for DB[a,h]A, I[1,2,3cd]P and B[ghi]P. The solvent delay time was 3 min (to bypass the solvent peak). For all the investigated analytes the corresponding ion ratios were used for confirmation purposes. Signal acquisition and data processing were performed using the Agilent MSD Chemstation 
(G1701EA, Agilent Technologies).

\subsection{Preparation of NF-PDA}

First of all, nickel foams (cutted by an art knife) were degreased with acetone for $20 \mathrm{~min}$, and then carefully cleaned with $2 \mathrm{M} \mathrm{HCl}$ solution in an ultrasound bath for $30 \mathrm{~min}$ in order to remove the $\mathrm{NiO}$ layer on the surface, and finally rinsed with de-ionized water and absolute ethanol, dried at room temperature.

Next, $200 \mathrm{mg}$ of NFs were added into a dilute aqueous solution of dopamine ( $2 \mathrm{mg}$ of dopamine per milliliter of $10 \mathrm{mM}$ tris, $\mathrm{pH} 8.5$ ). The solution was mechanically stirred for $12 \mathrm{~h}$ at room temperature to form a layer of polydopamine on the surface of NF, because dopamine could selfpolymerize in alkaline solution on virtually any surfaces [27]. The obtained black NF-PDA were washed with pure water for 5 times to remove the unreacted dopamine and dried at $50{ }^{\circ} \mathrm{C}$.

\subsection{Extraction procedure}

A piece of NF-PDA $(40 \mathrm{mg}$ ) sorbent was added into $100 \mathrm{~mL}$ water sample or PAHs-spiked water sample. After stirring for $5 \mathrm{~min}$ to accelerate the dispersion of the sorbent in solution, the NF-PDA was taken out rapidly from the solution by a forceps after adsorption equilibrium. Then an aliquot of $1.0 \mathrm{~mL}$ toluene was introduced to desorb the captured PAHs from the NF-PDA by sonication for $15 \mathrm{~s}$. Finally, $1 \mu \mathrm{L}$ of the extract was was injected into GC-MS system for analysis. The whole procedure is shown in Fig.1.

\section{Results and discussion}

\subsection{Characterization of NF-PDA}

The prepared NF-PDA was characterized with SEM, EDX and XPS. Figure 2 shows the SEM images of the 3D architectures of Ni foam and NF-PDA. SEM investigation showed that the naked Ni foam exhibited a $3 \mathrm{D}$ network-like structure composed of numerous pores, and the average pore diameter was about $220 \mathrm{~nm}$ (Fig.2a). After being functionalized with PDA, the colour of Ni foam turns from silver gray to black, and the pore diameter was smaller because of the coat (Fig.2b). The EDS spectrum of the Ni foam and NF-PDA film sample (Fig.S1) indicates the presence of $\mathrm{C}$ and $\mathrm{O}$ except of $\mathrm{Ni}$ on the surface of NF-PDA.

To determine the chemical composition and to further affirm the modification on the surface of NF-PDA, the X-ray photoelectron spectroscopy analysis is employed. Ni was not detected by XPS, which prove that dopamine thoroughly coated on the surface. The $\mathrm{C} 1 \mathrm{~s}, \mathrm{~N} 1 \mathrm{~s}$ 
and $\mathrm{O} 1 \mathrm{~s}$ deconvolution XPS spectra for NF-PDA are analyzed by curve fitting. As seen in Fig.S2, the $\mathrm{C} 1 \mathrm{~s}$ deconvolution spectra exhibit three components of the carbon bond at $284.4 \mathrm{eV}(\mathrm{C}-\mathrm{C} / \mathrm{C}-\mathrm{H}), 285.8 \mathrm{eV}(\mathrm{C}-\mathrm{N})$ and $286.5 \mathrm{eV}(\mathrm{C}=\mathrm{O})$. The $\mathrm{N}$ 1s deconvolution spectra exhibit the nitrogen bond at $399.6 \mathrm{eV}(\mathrm{N}-\mathrm{C} / \mathrm{N}-\mathrm{H})$. The $\mathrm{O} 1 \mathrm{~s}$ deconvolution spectra exhibit three components of the oxygen bond at $532.0 \mathrm{eV}(\mathrm{O}-\mathrm{C})$ and $532.9 \mathrm{eV}$ $(\mathrm{O}-\mathrm{H})$.

The specific surface area of the resultant NF-PDA was studied using Brurauer Emmerr Teller (BET) Procedure, giving a BET surface area of $4.437 \mathrm{~m}^{2} / \mathrm{g}$. The BET surface area is relatively small, which might be attributed to the relative large pore diameter of NF-PDA.

3.2. Optimization of extraction conditions

To obtain the highest extraction efficiency, extraction parameters were optimized, including the sorbent amount, type of desorption solvent, extraction time, water sample volume, elution volume, elution time, ionic strength and sample solution $\mathrm{pH}$. The optimization experiments were conducted using water sample spiked with $0.2 \mathrm{ng} / \mathrm{mL}$ of each PAH. Each experiment was performed in triplicate.

\subsubsection{Effect of amount of NF-PDA}

The effect of amount of NF-PDA was investigated with the amount varying from 10 to $120 \mathrm{mg}$. Fig. 3a demonstrates demonstrates that the recovery rate of almost all the 16 PAHs increase continuously with the increase of the sorbent amount from 10 to $40 \mathrm{mg}$. Further increasing the sorbent amount over $40 \mathrm{mg}$ results in no obvious increase of the recovery rate. These results indicated that $40 \mathrm{mg}$ of sorbents are sufficient to extract of PAHs from sample solution $(0.2 \mathrm{ng} / \mathrm{mL}, 100 \mathrm{~mL})$.

3.2.2 Effect of type of elution solvent 
An appropriate type of elution solvent is fundamental for extraction efficiency. Desorption of PAHs from NF-PDA sorbent was studied using $n$-hexane, dichloromethane and toluene. Results showed that $n$-hexane, dichloromethane and toluene are able to desorb target compound from the sorbents, and these three types of solvent are often used for esorption of PAHs. Herein, toluene was selected as elution solvent because it was more selective and effective due to its aromatic ring structure and its nonpolar feature. Also it yields higher analytical signals than n-hexane and dichloromethane (Fig. 3b). toluene proved to be the best choice for the extraction of the 16 PAHs and this behavior can be explained on the basis of the presence of $\pi-\pi$ interactions between the solvent and the aromatic rings of the analytes.

\subsubsection{Effect of extraction time and solution volume}

The bulk sorbent need sufficient contact with PAHs in water sample, which need adequate time. Fig.3c shows the effect of extraction time on the extraction efficiency. One can see that there is no obvious variation of the peak area when increasing the extraction time from 5-30 min. With this clarification, 5 min was sufficient to achieve satisfactory extraction efficiency for most PAHs.

To test the effect of solution volume on extraction efficiency of PAHs, the sample volume was varied from 10 to $100 \mathrm{~mL}$. As shown in Fig. 3d, the recoveries of all the PAHs do not decrease with sample volume increasing from 10 to $100 \mathrm{~mL}$. So, $100 \mathrm{~mL}$ was selected as sample volume.

3.2.4. Effect of elution volume and the elution time 
The elution volume and the elution time can affect the elution efficiency significantly. To study the effect of elution volume on the desorption, $0.5,1.0$ and $1.5 \mathrm{~mL}$ of were used as the eluent. The results in Fig. S3a show that $1.0 \mathrm{~mL}$ was sufficient to quantitatively elute 16 PAHs. Moreover, the effect of elution time on the recovery of PAHs was studied in the time range of 0.5-5 min, and the results are shown in Fig. S3b. It can be seen that the desorption kinetics was very quick and 1 min was enough for complete desorption of 16 PAHs. Finally, the elution time was set as $1 \mathrm{~min}$.

\subsubsection{Effect of ionic strength and sample solution $\mathrm{pH}$}

The effect of ionic strength on PAHs recoveries was examined by adjusting the salinity of sample solution with $\mathrm{NaCl}$ in the range of 0-300 $\mathrm{mM}$. The results indicated that the extraction efficiency of PAHs was independent of the ionic strength (Fig. S3c). We investigated the effect of solution $\mathrm{pH}$ on the recoveries of PAHs as the solution $\mathrm{pH}$ was ranged in 4-11. Fig.S3d shows that the recoveries of targets almost remained constant in the whole $\mathrm{pH}$ range. This result suggested the perfect stability of NF-PDA sorbents in both acid and alkaline sample solution.

Based on the above discussion, the optimal conditions of SPE with NF-PDA sorbent for PAHs are: $40 \mathrm{mg}$ NF-PDA, $100 \mathrm{~mL}$ solution volume, $5 \mathrm{~min}$ extraction time, $1.0 \mathrm{~mL}$ of toluene as the desorption solvent and 1 min of elution time.

\subsection{Stability of NF-PDA}

The stability of the NF-PDA sorbent was evaluated based on the repeatability of extraction efficiency using different batches of sorbent and the reusability of the sorbent.

The repeatability of the extraction was investigated using three different batches of NF-PDA with water sample spiked with $0.2 \mathrm{ng} / \mathrm{mL}$ of each PAH. The peak area and RSD values of 16 PAHs extracted with the 
three different batches of sorbents are listed in Table S1. The RSD values

are lower than $9.8 \%$, indicating a good reproducibility.

The regeneration of the sorbent was investigated with a random batch of NF-PDA as sorbent. The used NF-PDA (40 mg) was regenerated by rinsing it with $1 \mathrm{~mL}$ of toluene twice to make sure that no residul PAHs were remained. The regenerated sorbent was applied in SPE of water samples which were spiked with $0.2 \mathrm{ng} / \mathrm{mL}$ of each PAHs. As shown in Fig.S4, after 10 times of recycling, there is no significant change in the peak areas for each analyte extracted by the NF-PDA, indicating that the NF-PDA sorbents are stable and durable during SPE procedure with good reusability.

\subsection{Adsorption capacity of NF-PDA}

In order to study the adsorption capacity of a piece of NF-PDA sorbent, adsorption isotherm studies, solutions with different initial concentrations $(5-5000 \mathrm{ng} / \mathrm{mL})$. In the experiment, a piece of NF-PDA $(40 \mathrm{mg})$ and PAHs solution $(100 \mathrm{~mL})$ were mixed, and then the NF-PDA film was taken out rapidly from the solution by a forceps after adsorption equilibrium. The equilibrium concentration of PAHs was determined by GC-MS system. As shown in Fig.4, the calculated maximal adsorption capacities of Nap, Aceny, Acen, Flu, Phen, Ant, Fluo, Pyr, Chr, B[a]A, $\mathrm{B}[\mathrm{b}] \mathrm{F}, \mathrm{B}[\mathrm{k}] \mathrm{F}, \mathrm{B}[\mathrm{a}] \mathrm{P}, \mathrm{I}[1,2,3-\mathrm{cd}] \mathrm{P}, \mathrm{D}[\mathrm{a}, \mathrm{h}] \mathrm{A}$ and $\mathrm{B}$ [ghi]P on a piece of NFPDA were 192, 245, 162, 179, 198, 201, 325, 298, 225, 189, 185, 221, $107,125,198$ and $105 \mathrm{ng} / \mathrm{mg}$, respectively.

\subsection{Analytical figures of merit}

Under the optimal conditions mentioned above, a series of experiments with regard to the linearity (linear ranges and coefficient of determination), limit of detection (LOD), limit of quantification (LOQ), precision and enrichment factor (EF) were performed to validate the proposed method. In order to investigate the possible matrix effect on determination, the linearity of the proposed method was estimated by analyzing Ganjiang river water samples spiked with various concentrations of PAHs over a range of 10-1000, 25-1000 or 50-1000 ng/ L. The calibration curves were obtained by plotting the mean peak area versus sample concentration. The results are listed in Table 1. All the analytes show good linearity with coefficient of determination ranging from 0.9918 to 0.9994 .

The limit of detection (LOD) and the limit of quantification (LOQ) are calculated as the concentrations of the analytes at a signal-to-noise ratio $(\mathrm{S} / \mathrm{N})$ of 3 and 10 , respectively. Our results show that the LOD and 
LOQ values of the PAHs range from 2.3 (phen) to $16.5 \mathrm{ng} / \mathrm{L}(\mathrm{DB}[\mathrm{a}, \mathrm{h}] \mathrm{A})$, and from 7.7 to $55.0 \mathrm{ng} / \mathrm{L}$, respectively.

The EF of the method was calculated using the following equation:

$\mathrm{EF}=\left(\mathrm{V}_{s} / \mathrm{V} e\right) \times \mathrm{R} \%$

where $\mathrm{V}_{\mathrm{s}}$ is the sample volume, $\mathrm{V} e$ is the elution volume, and $\mathrm{R} \%$ is percent recovery. The EF were determined to be 91.4, 93.3, 96.1,95.8, 94.6, 97.5, 96.4, 92.4, 91.8, 93.5, 89.6, 90.6, 95.3, 92.4, 91.8 and 90.9 for Nap, Aceny, Acen, Flu, Phen, Ant, Fluo, Pyr, B[a]A, Chr, B[b]F, B[k]F, $\mathrm{B}$ [a]P, I[1,2,3-cd]P, DB[a,h]A and B[ghi]P, respectively.

Intra-day precision was evaluated by spiking Ganjiang River samples with appropriate amounts of PAHs at three different levels (100, 250 and $500 \mathrm{ng} / \mathrm{L}$ ). Six replicates were performed and analyzed on the same day. In order to determine reproducibility or inter-day precision, samples that were spiked with the same amount of PAHs, were analyzed in six consecutive days at three replicate levels. The intra and inter-day precisions were expressed as the percentage relative standard deviation (RSD\%) (Table 2). The intra-day precision was from 0.5 to $6.5 \%$ and the inter-day precision for the PAHs were below 9.3\%, illustrating the good repeatability achieved by the suggested procedure. These results imply that the proposed method can be applied to the analysis of real samples containing PAHs at trace level.

\subsection{Analysis of real water samples}

The method was applied to analyze Ganjiang river water and waste water samples under optimized conditions. Unspiked water samples and water samples spiked with 16 PAHs at two concentration levels (100 and $250 \mathrm{ng} / \mathrm{L})$ were analyzed by the proposed method ( $n=3)$. SPE-GC-MS chromatograms of Ganjiang river water and waste water spiked with PAHs are shown in Fig.5. The results are listed in Table S2. Nap and fluo were found in both Ganjiang river water and waste water samples, while 
phen was detected in waste water sample, too. The relative recoveries of PAHs at two concentration levels are in the range of 88.4-98.4\%, with RSDs within $7.3 \%$. These results imply that the established method can be applied to the analysis of PAHs at trace level in real samples.

\subsection{Comparison with other methods}

The proposed NF-PDA sorbent is compared with other SPE sorbents : $\mathrm{C}_{18}$ [34], multiwalled carbon nanotube (MWCNTs), Carbon- $\mathrm{Fe}_{3} \mathrm{O}_{4}$ [17], Carbon- $\mathrm{Fe}_{3} \mathrm{O}_{4}$ [35] and fluorenyl functionalized magnetic nanoparticles $\left(\mathrm{Fe}_{3} \mathrm{O}_{4} @ \mathrm{SiO}_{2} @ \mathrm{Flu}\right)$ [36] in Table 3. The comparison is performed on the basis of some operational (sorbent amount, extraction time and sample volume) and analytical properties (LOD, recovery and precision). Firstly, for comparison with sorbents for extraction of 16 PAHs, the NF-PDA needs less amount of sorbent than $\mathrm{C}_{18}$ [34] and MWCNTs [17], but more than Carbon- $\mathrm{Fe}_{3} \mathrm{O}_{4}$ [35]. Secondly, our SPE method is more convenient than traditional SPE, and as faster as the MSPE method. However, our extraction time is less than $\mathrm{C}_{18}$ and MWCNTs. Finally, the proposed method shows similar LODs and RSDs to the other three methods $[17,34,35]$, and the recoveries were comparable to others.

When compared to PDA-based SPE sorbents: Polydopamine-coated $\mathrm{Fe}_{3} \mathrm{O}_{4}$ nanoparticles $\left(\mathrm{Fe}_{3} \mathrm{O}_{4} @ \mathrm{PDA}\right)$ [29], magnetic $\mathrm{Fe}_{3} \mathrm{O}_{4} /$ polydopamine $\left(\mathrm{Fe}_{3} \mathrm{O}_{4} / \mathrm{PDA}\right)$ [30] and Polydopamine coated fiber (Fiber-PDA) [33]. .Firstly, for comparison with MSPE [29,30], the NF-based SPE method not need magnetic separation, and a forceps needed only to get out of the sorbent from sample solution instantly, which make the separation procedure faster and more convenient than MSPE. Secondly, It needs more amount of sorbent than $\mathrm{Fe}_{3} \mathrm{O}_{4} @ \mathrm{PDA}\left[29\right.$ ] and $\mathrm{Fe}_{3} \mathrm{O}_{4} / \mathrm{PDA}$ [30], but the extraction time of ours is shorter. For comparison with SPME [33], they are equally convenient, but the extraction time of NF- 
PDA based SPE is shorter. Fianlly, the proposed method shows similar RSDs to the other method, and the recoveries were comparable to others. In addition, our method applied to analyze 16 PAHs, while 1 or 6 PAHs analyzed by other three sorbents $[29,30,33]$. The $\pi-\pi$ stacking structure, $-\mathrm{OH}$ group, electron-deficient quinone structure and amine group on the surface of NF-PDA, all contributing to the selectivity to PAHs. Considering these results, the proposed sorbent is a sensitive, efficient, convenient and reliable material for the pre-concentration of trace PAHs.

\section{Conclusions}

In this work, a new sorbent based on PDA coated 3D NF was introduced for the enrichment of 16 U.S. EPA priority PAHs. The merits of the proposed SPE-GC-MS method can be concluded as the good adsorption toward PAHs due to the $\pi-\pi$ interactions of PDA moiety, the fast separation ability due to the clumpy sorbents, and the high stability. As a result, both the extraction and GC-MS analysis procedure were rapid. But there exist some deficiencies. As a bulk sorbent, the contact area was not large enough, which lead to low loading sample volume. On the other hand, the pore diameters of NF on the adsorption efficiency need to be researched in detail in the future.

The proposed method was applied in the analysis of Ganjiang river water and waste water samples. Nap and fluo were found in both Ganjiang river water and waste water samples, while phen was detected in waste water sample, too. The developed method is with great potential in the analysis of PAHs from aqueous samples.

\section{Acknowledgements}

We gratefully acknowledge the National Science Foundation of China (Grants No. 21175038, 21235002), the National Basic Research

Program of China (Grant No. 2009CB421601), and the Science and

Technology Support Program of Jiangxi Provincial Science and 
Technology Agency (Grant No. 20112BBG70021) for financial support.

\section{References}

[1] R. Ravindra, R. Sokhi, R. Van Grieken, Atmospheric polycyclic aromatic hydrocarbons: source attribution, emission factors and regulation, Atmos. Environ. 42 (2008) 2895-2921.

[2] R. E. Laflamme, R. A. Hites, The global distribution of PAH in recent sediments, Geochim. Cosmochim. Acta 42 (1978) 289-303.

[3] F. P. Perera, Environment and cancer: who are susceptible? Science 278 (1997) 1068-1073.

[4] U.S. EPA, Compendium of methods for the determination of toxic organic compounds in ambient air, in: Compendium Method TO-13A, Environmental Protection Agency, U.S. Federal Register, 1999, pp.1-42.

[5] Y. Liu, J. Shen, Z. Chen, N. Ren, Y. Li, Distribution of polycyclic aromatic hydrocarbons in surface water and sediment near a drinking water reservoir in Northeastern China, Environ. Sci. Pollut. Res. 20 (2013) 2535-2545.

[6] K. Kannan, B. Johnson-Restrepo, S. S. Yohn, J. P. Giesy, D. T. Long, Spatial and temporal distribution of polycyclic aromatic hydrocarbons in sediments from Michigan inland lakes. Environ. Sci. Technol. 39 (2005) 4700-4706.

[7] U.S. Environmental Protection Agency, Method 1654, in: Revision A. PAH Content of Oil by HPLC/UV, U.S. Environmental Protection Agency, 1992 (EPA Publication EPA-821-R-92-008) http://water.epa.gov/scitech/methods/cwa/oil/ upload/2007 0710 methods method oil 1654.pdf.

[8] U.S. Environmental Protection Agency, Polynuclear aromatic hydrocarbons, in: Method 8310, U.S. Environmental Protection Agency, 1986 (EPA SW-846) http://www.epa.gov/osw/hazard/testmethods/sw846/pdfs/8310.pdf.

[9] M. Olson, J. Iverson, E. Furlong, M. Schroeder, USGS Water Resources Investigations Report 03-4318, 2004, http://nwql.usgs.gov/rpt.shtml?WRIR-034318

[10] Z. Wang, S.A. Stout (Eds.), Oil Spill Environmental Forensics: Fingerprinting 
and Source Identification, 1st ed., Academic Press, New York, 2007.

[11] J.I. Cacho, N. Campillo, P. Viñas, M. Hernández-Córdoba, Use of headspace sorptive extraction coupled to gas chromatography-mass spectrometry for the analysis of volatile polycyclic aromatic hydrocarbons in herbal infusions, J. Chromatogr. A 1356 (2014) 38-44.

[12] L.D. Gratz, S.T. Bagley, D.G. Leddy, J.H. Johnson, C. Chiu, P. Stommel, Interlaboratory comparison of HPLC-fluorescence detection and GC/MS: analysis of PAH compounds present in diesel exhaust,J. Hazard. Mater. 74 (2000) 37-46.

[13] Q. L. Li, X. Wang, X. F. Chen, M. L. Wang, R. S. Zhao, In situ hydrothermal growth of ytterbium-based metal-organic framework on stainless steel wire for solid-phase microextraction of polycyclic aromatic hydrocarbons from environmental samples, J. Chromatogr. A 1415(2015)11-19.

[14] G. Purcaro, M. Picardo, L. Barp, S. Moret, L. S. Conte, Direct-immersion solid-phase microextraction coupled to fast gas chromatography mass spectrometry as a purification step for polycyclic aromatic hydrocarbons determination in olive oil, J. Chromatogr. A 1307(2013)166-171.

[15] H.Y. Fu, D.Q. Zhu, In situ hydrothermal grown silicalite-1 coating for solidphase microextraction, Anal. Chem. 84 (2012) 2366-2372

[16] Z.G. Shi, H.K. Lee, Dispersive Liquid-Liquid Microextraction Coupled with Dispersive $\mu$-Solid-Phase Extraction for the Fast Determination of Polycyclic Aromatic Hydrocarbons in Environmental Water Samples, Anal. Chem. 82 (2010) 1540-1545.

[17] L. Guo, H. K. Lee, Development of multiwalled carbon nanotubes based micro-solid-phase extraction for the determination of trace levels of sixteen polycyclic aromatic hydrocarbons in environmental water samples, J. Chromatogr. A 1218 (2011) 9321-9327.

[18] H. Zhang, W. P. Low, H. K. Lee, Evaluation of sulfonated graphene sheets as sorbent for micro-solid-phase extraction combined with gas chromatography-mass spectrometry, J. Chromatogr. A 1233 (2012) 16-21. 
[19] S.W. Xue, M.Q. Tang, L. Xu, Z.G. Shi, Magnetic nanoparticles with hydrophobicity and hydrophilicity for solid-phase extraction of polycyclic aromatic hydrocarbons from environmental water samples, J. Chromatogr. A 1411(2015) 9-16.

[20] L. Hao, C. Wang, Q. H. Wu, Z. Li, X. H. Zang, and Z. Wang, Metal-organic framework derived magnetic nanoporous carbon: novel adsorbent for magnetic solid-phase extraction, Anal. Chem. 86 (2014) 12199-12205.

[21] J. Yang, J. Y. Li, J.Q. Qiao, H. Z. Lian, H. Y. Chen, Solid phase extraction of magnetic carbon doped $\mathrm{Fe}_{3} \mathrm{O}_{4}$ nanoparticles, J. Chromatogr. A 1325(2014) $8-15$.

[22] Y. Cai, Z. H. Yan, N. Y. Wang, Q. Y. Cai, S. Z. Yao, Preparation of naphthyl functionalized magnetic nanoparticles for extraction of polycyclic aromatic hydrocarbons from river waters, RSC Adv. 5(2015) 56189-56197.

[23] B. Socas-Rodríguez, J. Hernández-Borges, P. Salazar, M. Martín, M. Rodríguez-Delgado, Core-shell polydopamine magnetic nanoparticles as sorbent in micro-dispersive solid-phase extraction for the determination of estrogenic compounds in water samples prior to high-performance liquid chromatography-mass spectrometry analysis, J. Chromatogr. A 1397 (2015) $1-10$

[24] T. A. Schaedler, A. J. Jacobsen, A. Torrents, A. E. Sorensen, J. Lian, J. R. Greer, L. Valdevit, W. B. Carter, Ultralight metallic microlattices, Science 334 (2011) 962-965.

[25] H. W. Ge, C. X. Wang, L. W. Yin, Hierarchical $\mathrm{Cu}_{0.27} \mathrm{Co}_{2.73} \mathrm{O}_{4} / \mathrm{MnO}_{2}$ nanorod arrays grown on 3D nickel foam as promising electrode materials for electrochemical capacitors, J. Mater. Chem. A, 3 (2015) 17359-17368.

[26] Y. Bai, W.Q. Wang, R.R. Wang, J. Sun, L. Gao, Controllable synthesis of 3D binary nickel-cobalt hydroxide/graphene/nickel foam as a binder-free electrode for high-performance supercapacitors, J. Mater. Chem. A, 3 (2015) 12530-12538.

[27] H. Lee, S.M. Dellatore, W.M. Miller, P.B. Messersmith, Mussel-inspired surface chemistry for multifunctional coatings, Science 318 (2007) 426-430. 
[28] Q. Ye, F. Zhou, W. Liu, Bioinspired catecholic chemistry for surface modification, Chem. Soc. Rev. 40 (2011) 4244-4258.

[29] Y.R. Ma, X.L. Zhang, T. Zeng, D. Cao, Z. Zhou, W.H. Li, H.Y. Niu, Y.Q. Cai, Polydopamine-coated magnetic nanoparticles for enrichment and direct detection of small molecule pollutants coupled with MALDITOF-MS, ACS Appl. Mater. Interfaces 5 (2013) 1024-1030.

[30] Y.X. Wang, S.H. Wang, H.Y. Niu, Y.R. Ma, T. Zeng, Y.Q. Cai, Z.F. Meng, Preparation of polydopamine coated $\mathrm{Fe}_{3} \mathrm{O}_{4}$ nanoparticles and their application for enrichment of polycyclic aromatic hydrocarbons from environmental water samples, J. Chromatogr. A, 1283 (2013) 20-26

[31] B. Socas-Rodríguez, J. Hernández-Borges, P. Salazar, M. Martín, M. Á. Rodríguez-Delgado, Core-shell polydopamine magnetic nanoparticles as sorbent in micro-dispersive solid-phase extraction for the determination of estrogenic compounds in water samples prior to high-performance liquid chromatography-mass spectrometry analysis, J. Chromatogr. A, 1397 (2015) $1-10$

[32] Z.Z. Huang, H. K. Lee, Study and comparison of polydopamine and its derived carbon decorated nanoparticles in the magnetic solid-phase extraction of estrogens, J. Chromatogr. A, 1414 (2015) 41-50

[33] J.J. Feng, M. Sun, J.B. Li, L.L. Xu, X. Liu, S.X. Jiang, polydopamine supported preparation method for solid-phase microextraction coatings on stainless steel wire, J. Chromatogr. A, 1218 (2011) 3601-3607.

[34] E. Martinez, M. Gros, S. Lacorte, D. Barceló, Simplified procedures for the analysis of polycyclic aromatic hydrocarbons in water, sediments and mussels, J. Chromatogr. A, 1047 (2004) 181-188.

[35] L. Bai, B. Mei, Q. Z. Guo, Z. G. Shi, Y. Q. Feng, Magnetic solid-phase extraction of hydrophobic analytes in environmental samples by a surface hydrophilic carbon-ferromagnetic nanocomposite, J. Chromatogr. A, 1217 (2010) 7331-7336.

[36] Y. Cai, Z. H. Yan, M. NguyenVan, L. J. Wang, Q.Y. Cai, Magnetic solid phase extraction and gas chromatography-mass spectrometrical analysis of sixteen polycyclic aromatic hydrocarbons, J. Chromatogr. A, 1406 (2015) 40-47. 


\section{Figure Captions}

Fig.1 Scheme of procedure of the analysis for PAHs by using NF-PDA

Fig.2 SEM image of the Ni foam (a) and NF-PDA (b) film sample, and the insets are photos of Ni foam (a) and NF-PDA (b).

Fig.3 Effect of (a) sorbent amount, (b) type of desorption solvent, (c) extraction time, (d) sample volume on the peak areas or recoveries of PAHs.

Fig.4 Adsorption isotherms for PAHs on NF-PDA.

Fig.5 GC-MS chromatograms of SPE of water samples: (a) Ganjiang river water sample spiked with $250 \mathrm{ng} / \mathrm{L}$ of each analyte; (b) non spiked Ganjiang river water and (c) non spiked waste water sample. Peaks: (1) Nap, (2) Aceny, (3) Acen, (4) Flu, (5) Phen, (6) Ant, (7) Fluo, (8) Pyr, (9) B[a]A, (10) Chr, (11) B[b]F, (12) B[k]F, (13) $\mathrm{B}[\mathrm{a}] \mathrm{P},(14) \mathrm{I}[1,2,3-\mathrm{cd}] \mathrm{P},(15) \mathrm{DB}[\mathrm{a}, \mathrm{h}] \mathrm{A}$ and (16) B [ghi]P.

Table captions

Table 1 SPE-GC/MS analytical figures of merit for the16 PAHs.

Table 2 The precision of the method.

Table 3 Comparison of the proposed method with other procedures previously reported for analysis of PAHs 


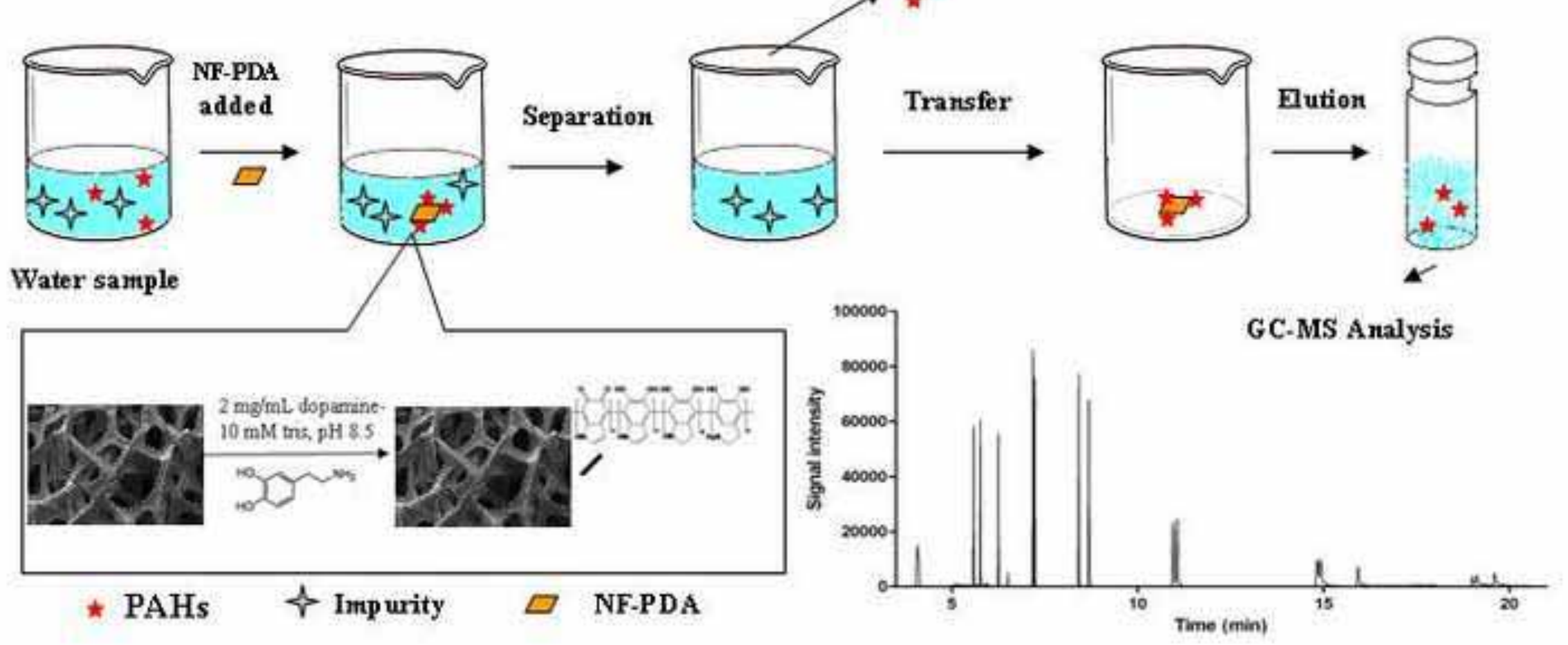




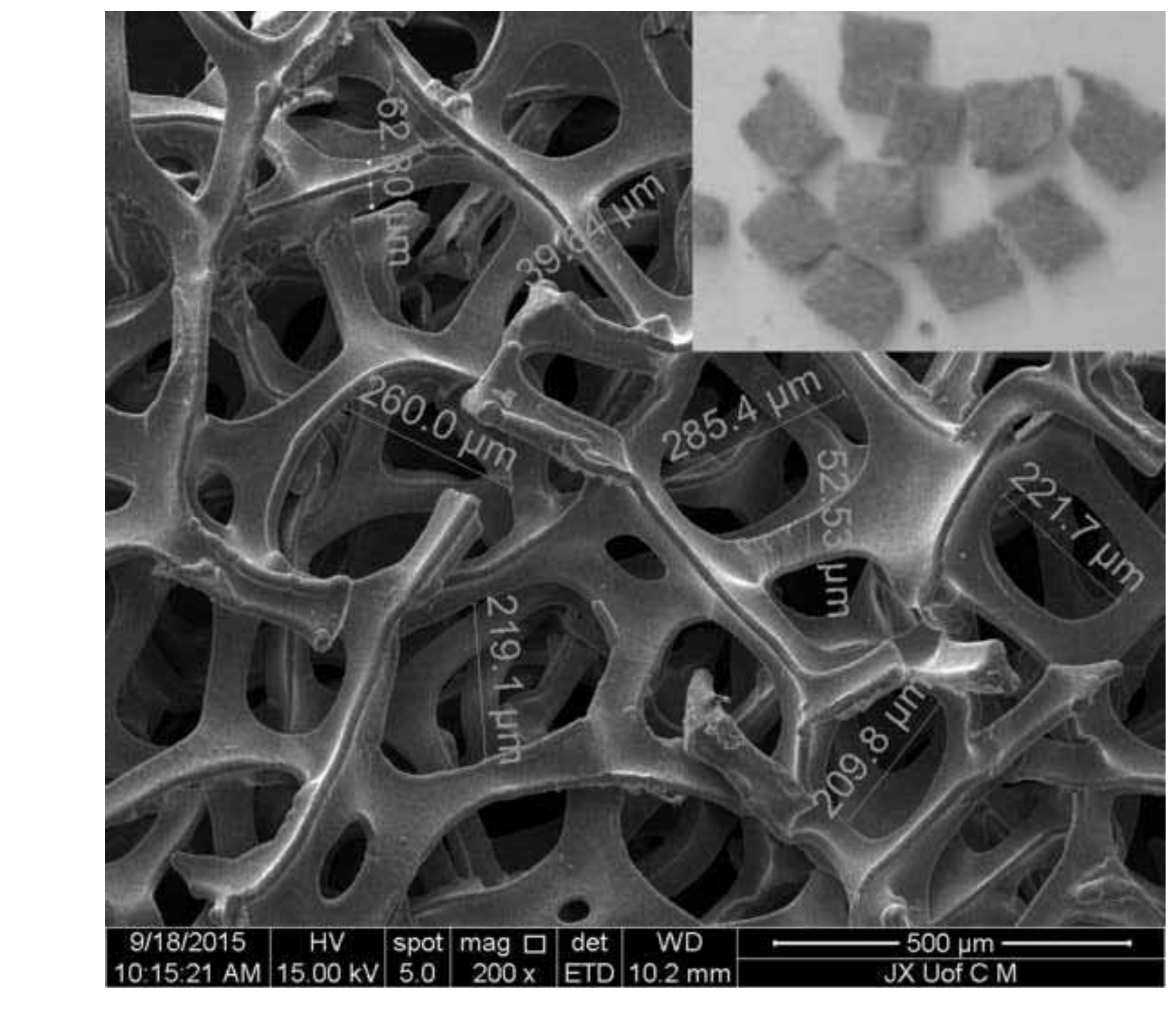

.
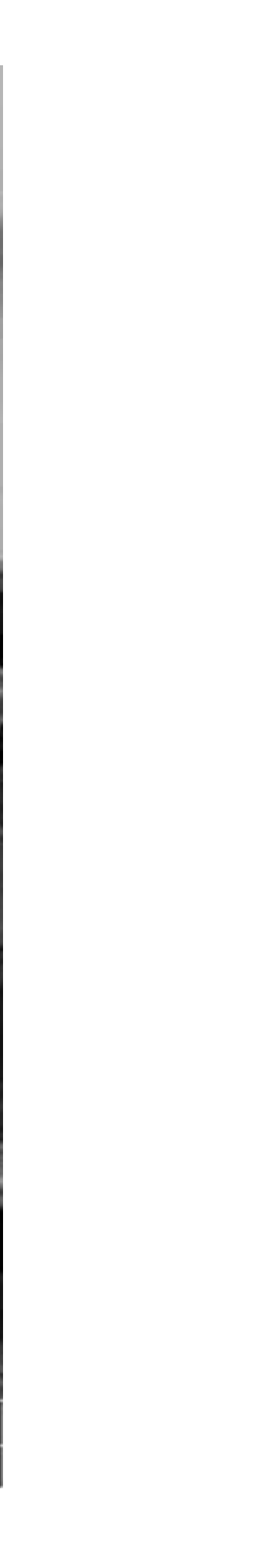


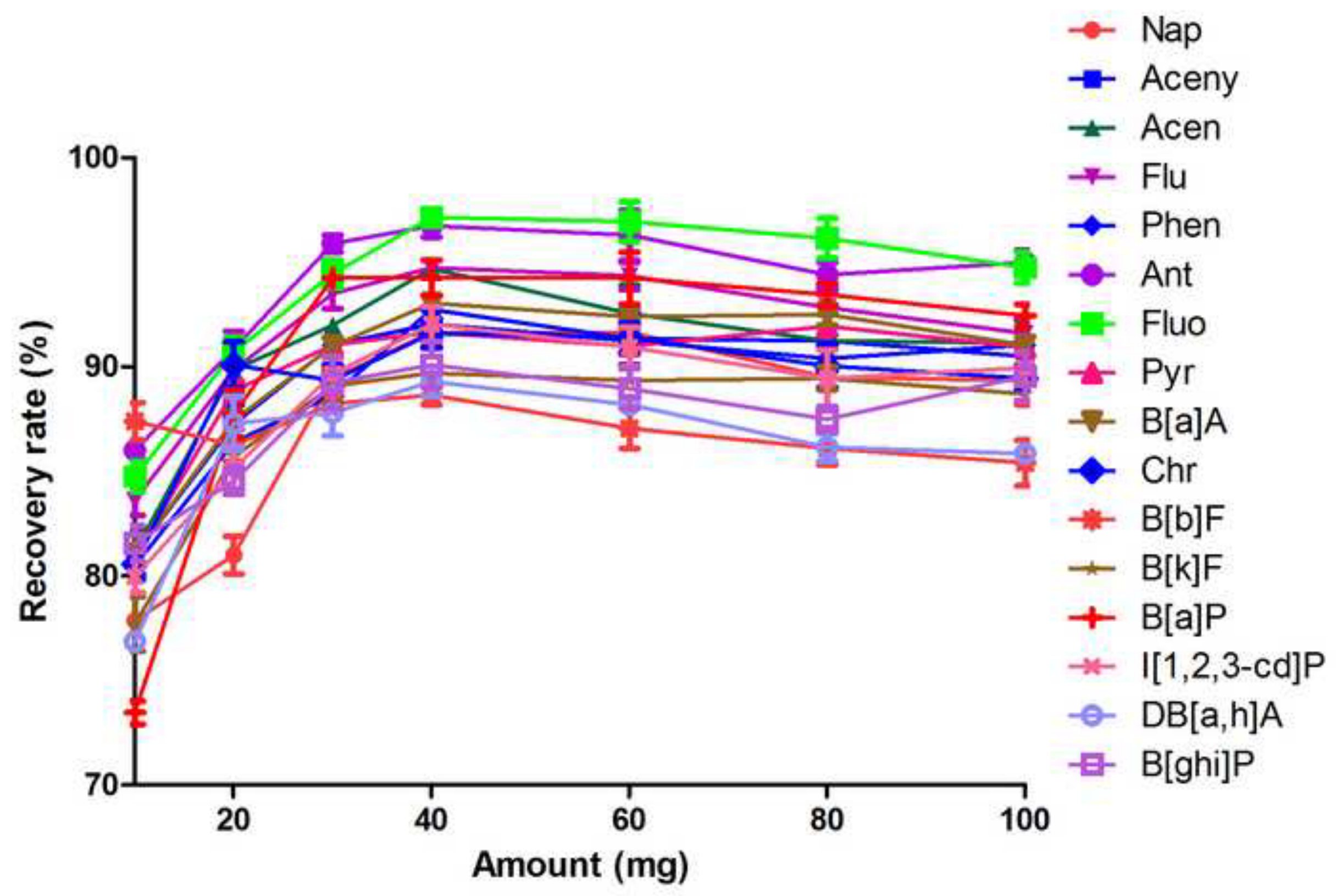




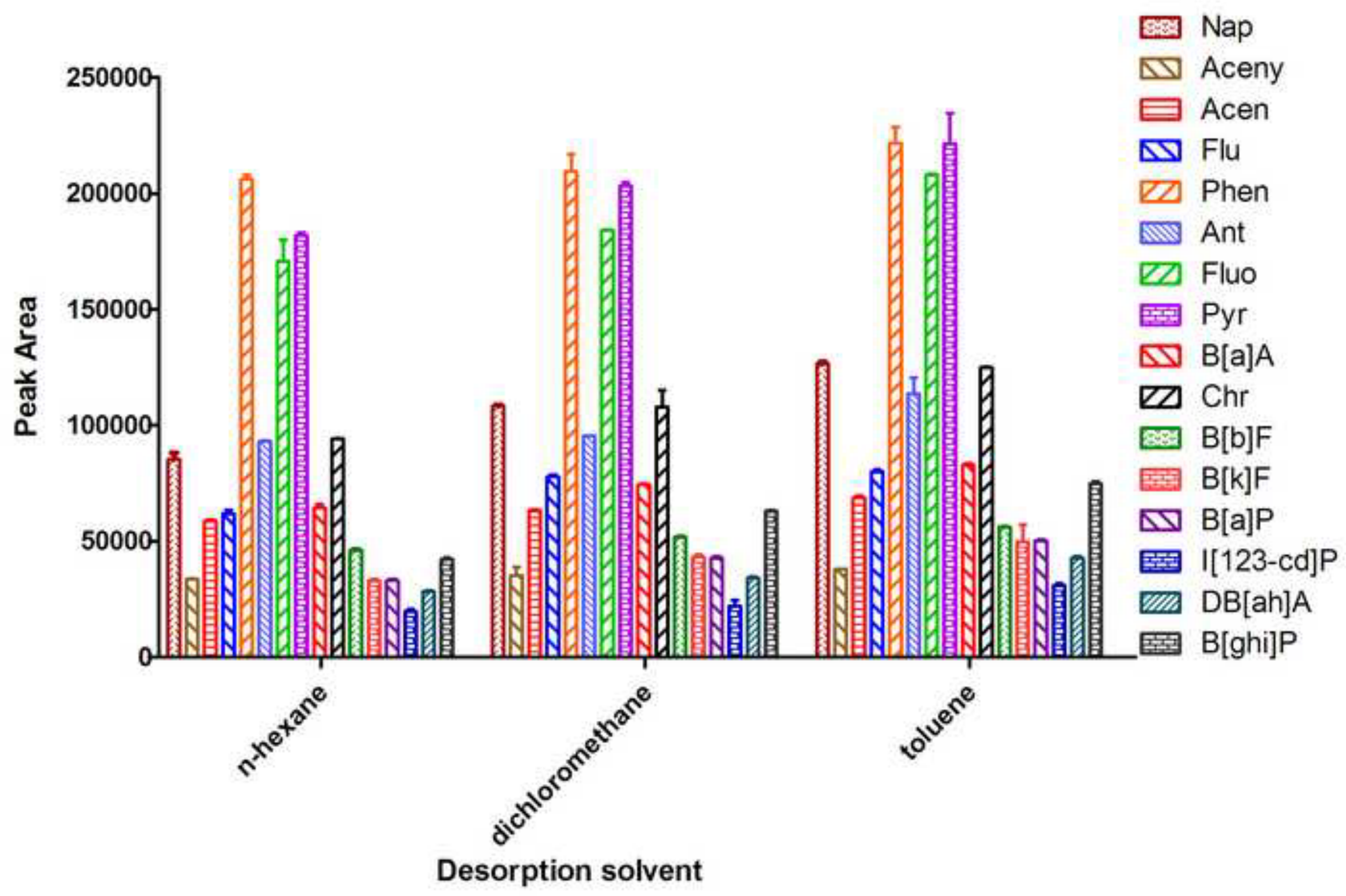




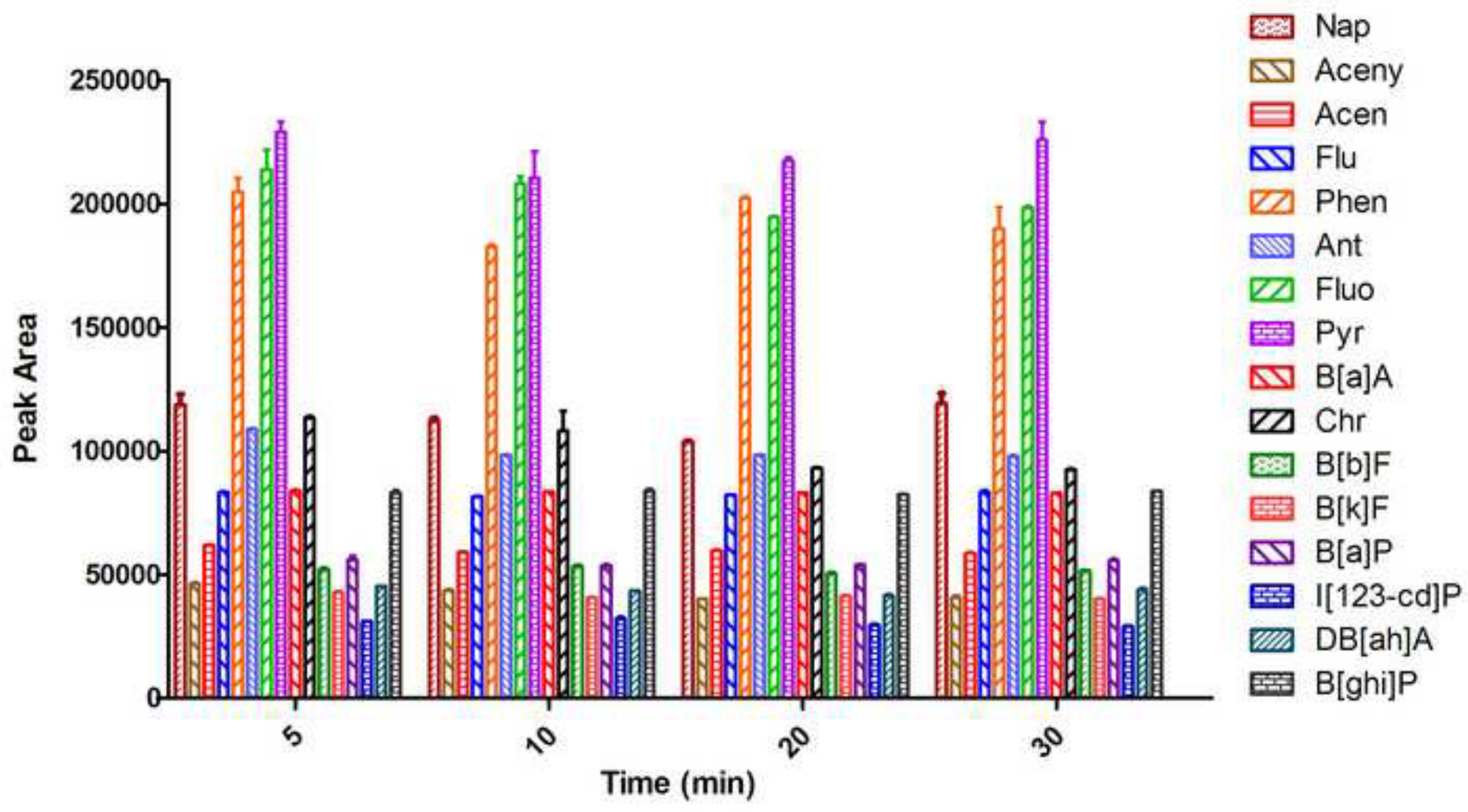




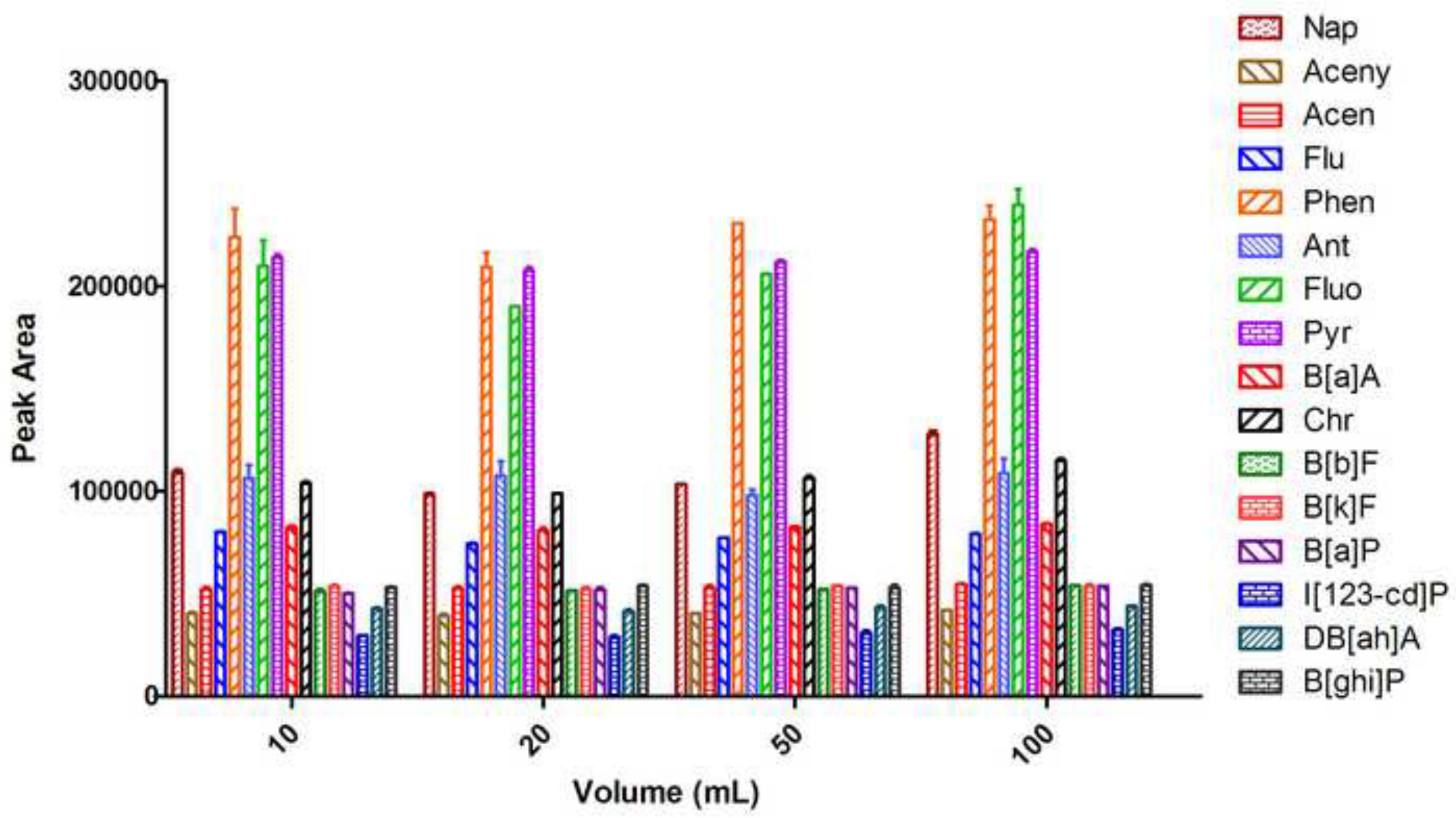




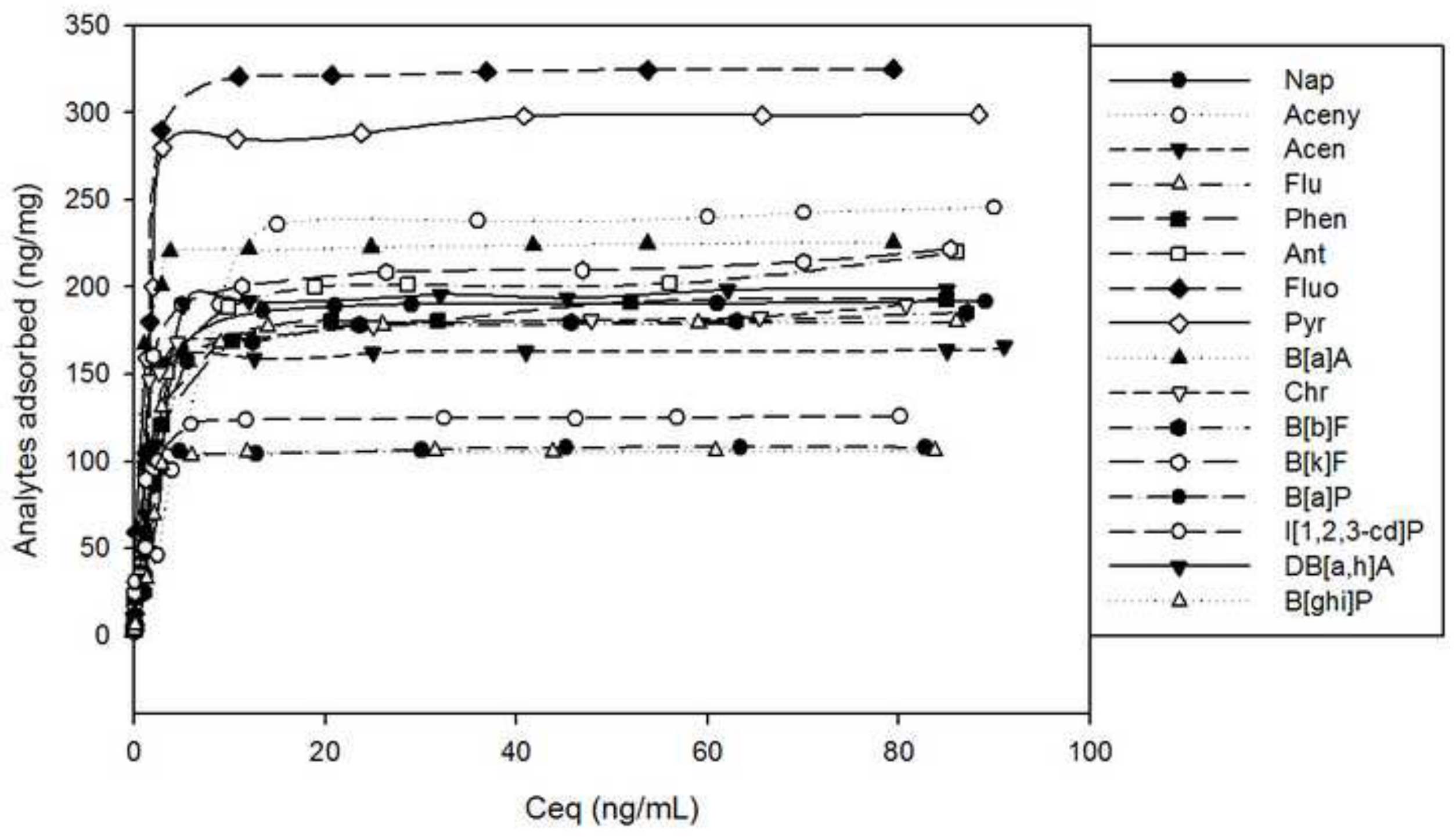




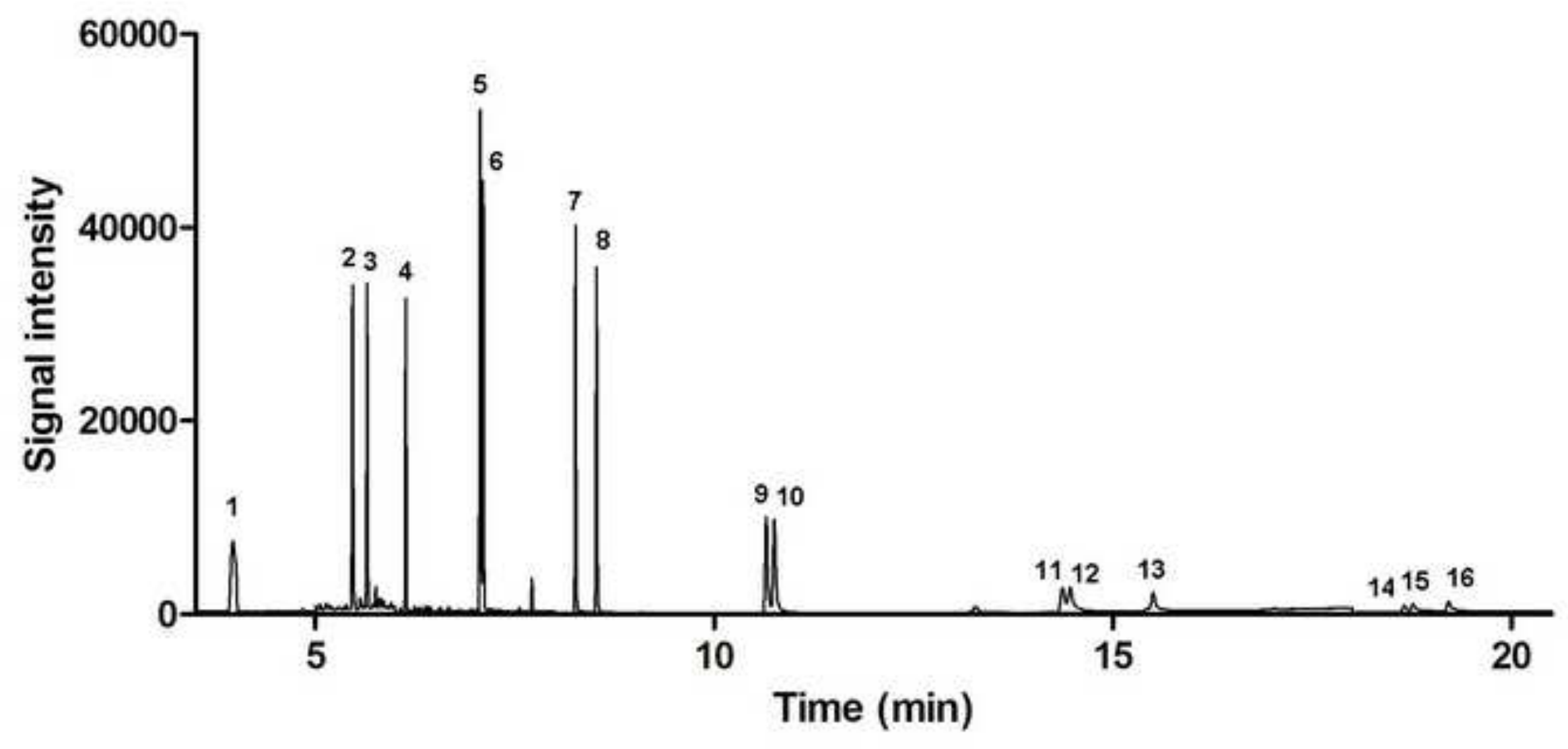




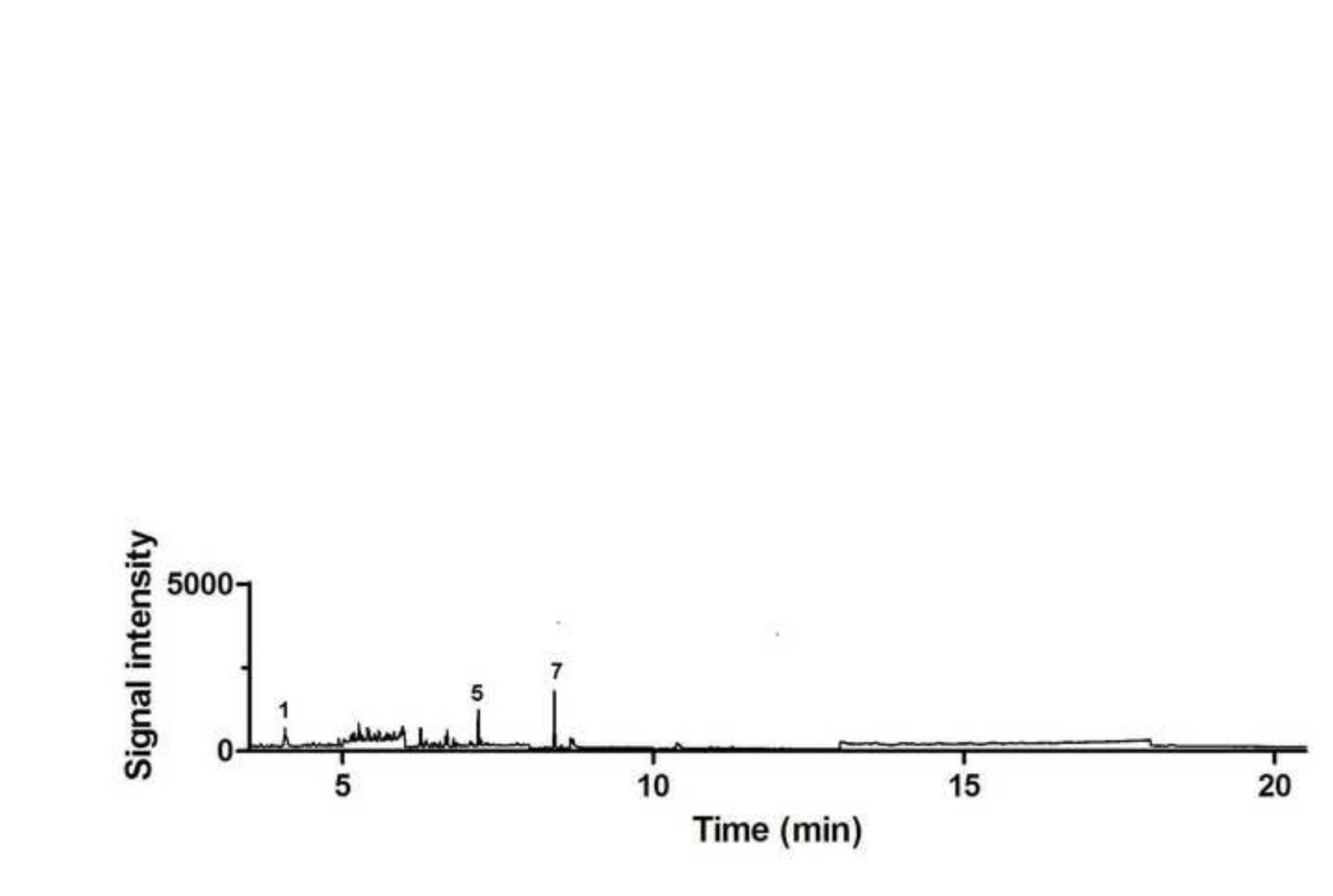

Time (min) 